\title{
EAER: An Alternative and Effective Remineralization non- invasive method in Restoration of carious tooth
}

\author{
Kamala C1, ${ }^{*}$, Gopala Krishna Ganta ${ }^{2}$, Anoosha Manda ${ }^{3}$ \\ ${ }^{1}$ Practitioner, Shankar Multispecialty Hospital, Kakinada, East Godavari, 533003, Andhra Pradesh, India. \\ 2Former Undergraduate Student, Vishnu Dental College, Bimavaram, West Godavari, 534202, Andhra \\ Pradesh, India. \\ ${ }^{3}$ Senior Lecturer, Department of Orthodontics, Vishnu Dental College, Bhimavaram, West Godavari, \\ 534202, Andhra Pradesh, India.
}

I N F O R M A T I O N

\section{Article History}

Received 20 April 2019

Received revised

30 April 2019

Accepted 07 May 2019

Available online 15 May 2019

\section{K E Y W O R D S}

\section{EAER}

Demineralization

Remineralization

\section{A B S T R A C T}

This article deals with the newer technologies that are used in the remineralization procedure of tooth thus avoiding usage of drills on the tooth structure and preserving the enamel as a whole. A number of finer and newer technologies are being developed so that procedures can be more friendly and acceptable to both the dentist as well as the patient, these newer strategies are also concerned in preserving the tooth structure as a whole without the introduction off any foreign material, thus increasing the life as well as the strength of the tooth structure. This paper gives an ephemeral information on various newer strategies of remineralization with more emphasis on the most recently introduced remineralization technique such as electrically accelerated and enhanced remineralization technique (EAER).

\section{Introduction}

Dental caries is the one of the most common dental issues among the various populations irrespective of age, gender and geographic regions. The treatment available is simple cost effective yet many people neglect it due to dental phobia and lack of dental treatment awareness. The deep hidden fear for the drills and injections has led to ignore the treatment by the patients. Various dentists have revealed that in order to control the anxiety of the patients, they sometimes have to advice sedation [1]. However, this treatment does not only remove infected part but also part of the healthy tooth structure in order to create room for restorative material and also to provide proper retention with it. Hence, it is necessary to explore an alternative treatment modality which should eliminate the phobia among the patients, less technique sensitive. In addition, that should also prevent the recurrent caries and strengthen the natural tooth. This article provide an overview of the various newer techniques developed on remineralisation procedures and gives more

Correspondence: *Corresponding author Email Address: kamala1995@gmail.com. How to cite this article: Kamala C, Gopala Krishna Ganta, Anoosha Manda. EAER: An Alternative and Effective Remineralization Method. Int J Dent Mater 2019;1(1):36-39. DOI: http://dx.doi.org/10.37983/IJDM.2019.1105 
emphasis on mechanism, advantages and disadvantages of Electrically accelerated and enhanced remineralisation (EAER) method, so that this newer technique could be made more familiar to practitioners.

\section{Pathophysiology of cavity formation}

Dental cavities are not mere a stationary process of tooth loss but rather it's a dynamic process; there is constant conflict between the demineralization process and remineralization process. Various acids are produced by the microbial flora during the metabolism of the carbohydrates that results in the drop in $\mathrm{pH}$. This leading to the dissolution of minerals such as calcium and phosphates in their ionic forms from the tooth surface as well as sub-surface leading to demineralization of the tooth and ultimately leading to cavity formation [2]. The various factors that are responsible for demineralisation that ultimately leads to caries formation are cariogenic bacteria such as streptococcus mutants, lactobacillus etc., fermentable carbohydrates such as sugar and the saliva. The remineralisation factors are antibacterial agents, saliva and remineralizing ions [3]. The remineralizing action takes place when minerals like fluoride are introduced in the surface of enamel, where it absorbs and holds minerals and fluoride which are present in the plaque and help in the remineralisation of the surface that is lost due to cariogenic activity [2]. The thorough detailed pathophysiology has allowed us to incorporate a more regenerative procedure rather than just curative. Various regenerative procedures are given in the subsequent sections.

\subsection{Dentin phosphoprotein 8DSS peptides}

The phosphorprotein is the non-collagenous extracellular element of the dentin [4]. The human dentin phosphoprotein peptides (DPP) have proven to have aspartate-serine-serine nucleotides which help in the production of hydroxy apatite (HA) crystals. The 8DSS peptide have two mineral binding sites that attaches to both free calcium, phosphate ions and even to HA crystals. When these peptides are applied to the enamel surface prevents the dissolution of these ions and further helps in attracting calcium and phosphate ions. Thus, helps in remineralization[5]. This procedure is still in laboratory phase and no commercial products are yet available in market.

\subsection{P11-4 peptides}

The fibilliar matrix has the ability to attract the calcium channels towards itself. It also acts as a new productive site for HA crystals, these peptides selfassemble in hierarchical 3-dimensional way at the site of lesion in response to high acidic $\mathrm{pH}$ of the surrounding. When low viscosity isotropic P11-4 is applied to the enamel surface it transforms into an elastomeric gel, thus leading to restoration of the fibrillar matrix and ultimately enabling the lost tooth structure to remineralize [6].

\subsection{Leucine-rich amelogeninpeptides}

This technique proves to be more successful in invitro studies. The invitro studies have proved that the tooth that is treated with amino acids containing leucine rich amelogenin peptide not only reduced the depth of the lesion but also provided the required elements for the growth of enamel peptides, hence helping in remineralization [7]. This amelogenin peptide only contains the $\mathrm{C}$-terminal and $\mathrm{N}$ - terminal of the parent protein. These domains are seen to be responsible for the binding and linear growth of the mineral content.

\subsection{Poly(amino amine) dendrimers}

These are also called as artificial proteins as they mimic the matrix that is required for mineralization. The organic crystal that is formed is known to be formed through poly amino amine dendrimers template has the exact content shape and size as that of intact enamel, in fact the rods even tend to align parallel to the existing HA crystals [8,9].

\subsection{Nanohydroxyapatite}

The synthetic hydroxapatite have shown to be best in terms of biocompatibility [10], due to its nano size it can be compactly packed, increases the surface area to bind with other particles and also fills the tiny holes. The exact mechanism behind remineralization with the help of nanohydroxyapatitie (nHA) is still unknown, many researchers explain that nHA promotes remineralization by acting as a layer of enamel around the defective area. Other researchers suggest that nHA crystals acts as reservoir of calcium and phosphate ions, thus these ions are present in supersaturated state thus enabling remineralization and suppressing demineralization [11]. 
2.6. Electrically accelerated and enhanced remineralization

Among the remineralization procedures discussed from section 2.1 to 2.5 , electrically accelerated and enhanced remineralization (EAER) has received more attention among the researchers and it was introduced by the researchers at King's college, London. EAER is based on the theory of iontophoresis, which is a widely used and accepted method for transdermal and ocular drug delivery $[12,13]$. In iontophoresis, the charged particles get attracted towards the opposite electrode until and unless the electric field is lowered. EAER is a process that aims at enhancing the uptake of the various remineralization factors towards the damaged tooth, which results in shifting of the equilibrium from demineralization state to remineralization state. Therefore, the tooth is protected from decay and is restored well without any drill and fill $[1,12]$.

The mechanism behind the EAER is quite simple and unique. Figure 1 describes the various steps that are involved in restoring caries lesions to the equivalent of healthy enamel using EAER. The tooth surface is cleaned and conditioned, initially. Then, the actual remineralization process takes place by increasing the concentration of all the natural minerals at the site which is to be repaired, with the help of electric field from a custom-made dental device. This helps in enhancing the natural repair of the damaged/infected tooth structure [14].

Pitts NB and Wright JP (2018) experimented with gold nanoparticles instead of remineralizing agents as they are easy to detect and locate the exact location in the lesion. They observed the presence of gold nanoparticles almost into the entire lesion, that is considered as activated sites with lesion and also in areas of organic substances which prevented surface activation.[14]

\subsubsection{Advantages of EAER [1]}

- Elimination of phobia needles, sounds, smell etc.,

- No loss of any health tooth structure.

- No introduction of foreign materials into the body thus eliminating many allergic and rejection reactions.

- No requirement of refilling of restorations which is generally done to conventional materials after a period of time due to polymerisation shrinkage.

- User friendly for the dentist.
Cleaning \& Conditioning

(Remove all decayed material from lesion)

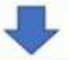

\section{Activation}

(Optimization of lesion surfaces for remineralization)

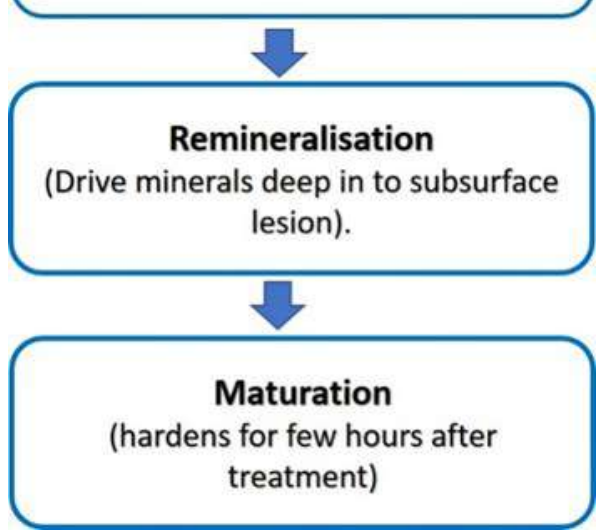

Figure 1. Steps involved in using EAER (Electrically Accelerated and Enhanced Remineralization) to restore caries lesions

- Teeth treated with EAER are stronger than those filled with fillings and even help in preventing further decay.

- Highly esthetic and has positive effect on patients.

- Repair the entire lesion.

- No damage to existing healthy tissues.

- Also helps in whitening the teeth, thus a better option than bleaching.

\subsubsection{Limitations of EAER}

- No clear evidence if this procedure could be used even in cases of pulpal involvement.

- Since biomimetic materials are to be used then can be more expensive than the conventional dental restorative materials.

- Less evidence of successful clinical trials.

\section{Conclusion}

The concept of remineralization is one of the most studied and researched topic since the early $20^{\text {th }}$ century, but yet even till day we still practice invasive form of dentistry, with developing technology it's time for a shift from invasive form to a non-invasive form of dentistry, EAER is one the most ideal way to practice non invasive methods as it only saves the tooth but also st- 
rengths it. The small "healing hand piece" placed on the damaged tooth eliminates the phobia associated with drill among the patients, even the electric current used is minimal, it's even less than that are used for electric pulp testing, thus making it not only just effective but one of the safest techniques. With these newer innovations the future dentistry looks promising and without drills.

\section{References}

1. Kakkar M. An alternative to filling and drilling EAER. Int J Res Health Allied Sci 2016;2(2):32-34.

2. Tondon, Textbook of Pedodontics, 2nd edition, published by Divyesh Arvind Kothari for Paras Medical Publisher, 2009:267-268.

3. Featherstone JD, Chaffee BW. The evidence for caries management by risk assessment (CAMBRA). Adv Dent Res 2018; 29: 9-14.

4. Hsu CC, Chung HY, Yang JM, Shi W, Wu B. Influence of 8DSS peptide on nano-mechanical behavior of human enamel. J Dent Res 2011; 90: 88-92.

5. Prasad M, Butler WT, Qin C. Dentin sialophosphoprotein in biomineralization. Connect Tissue Res 2010; 51: 404-417.

6. Kirkham J, Firth A, Vernals D, Boden N, Robinson $\mathrm{C}$, Shore RC, et al., Self-assembling peptide scaffolds promote enamel remineralization. J Dent Res 2007; 86: 426- 430 .

7. Bagheri GH, Sadr A, Espigares J, Hariri I, Nakashima S, Hamba $\mathrm{H}$, et al., Study on the influence of leucine-rich amelogenin peptide (LRAP) on the remineralization of enamel defects via micro-focus X-ray computed tomography and nanoindentation. Biomed Mater 2015;10(3): 035007.

8. Wu D, Yang J, Li J, Chen L, Tang B, Chen X, et al., Hydroxyapatite-anchored dendrimer for in situ remineralization of human tooth enamel. Biomaterials 2013; 34: 5036-5047.

9. Yang S, He H, Wang L, Jia X, Feng H: Oriented crystallization of hydroxyapatite by the biomimetic amelogeninnanospheres from self-assemblies of amphiphilicdendrons, Chem. Commun. 2011,47 (36): 10100-10102.

10. Hanning M, Hanning C. Nanomaterials in preventive dentistry. Nat Nanotechnol 2010; 5: 565-569.

11. Pepla E, Besharat LK, Palaia G, Tenore G, Migliau G. Nano-hydroxyapatite and its applications in preventive, restorative and regenerative dentis- try: A review of literature. Ann Stomatol (Roma) 2014; 5: 108-114.

12. Sarraf D, Lee DA. The role of iontophoresis in ocular drug delivery. J Occul Pharmocol 1994; 10 (1):69-81.

13. Prausnitz MR, Mitragotri S, Langer R. Current status and future potential of transdermal drug delivery. Nat Rev Drug Discov. 2004;3(2):115-24.

14. Pitts NB, Wright JP: Reminova and EAER: keeping enamel whole through caries remineralization. Adv Dent Res 2018; 29: 48-54. 